\title{
VARIACION MENSUAL DE LA COMPOSICION QUIMICA DEL MEJILLON PERNA PERNA (L.) CULTIVADO Y LAS CONDICIONES AMBIENTALES EN LA BAHIA EL GUAMACHE, ISLA DE MARGARITA, VENEZUELA
}

\author{
JOSE PINEDA Y AGUSTIN AGUADO
}

Centro de Investigaciones Científicas, Universidad de Oriente, Nueva Esparta, Venezuela

\begin{abstract}
Monthly variations in the chemical composition of the mussel (Perna perna) cultivated and the environment factors were studied in the Guamache Bay (Margarita Island. Venezuela). The study was carried out on mussels of $65.70 \mathrm{~mm}$ shell lenght and on a size that was monthly increased in $5 \mathrm{~mm}$ from $40.45 \mathrm{~mm}$ to $100-105 \mathrm{~mm}$. The weight of meat ranged from 8,05 to $10,87 \mathrm{~g}$, the weight of shell from 8,88 to $11,00 \mathrm{~g}$ and weight of the intervalve water from 2,64 to $6,83 \mathrm{~g}$. In both experiences the water fluctuated between 72,2 and $86,7 \%$ ashes from 2,25 to $3,58 \%$ and proteins between 9,10 and $16,05 \%$. The influence of the meteorological conditions on temperature and salinity was analized. The percentage of oxygen saturation was always over $80 \%$. The primary nutrients registered low values only the ammonia mean, reached 2,43 $\mu \mathrm{g} a \mathrm{t} / \mathrm{L}$ Chlorophyll " $\mathrm{a}$ " fluctuated between 0,25 and $4,50 \mu \mathrm{g} / \mathrm{L}$. The results showed that the variations in the chemical composition of mussels was independent of the size and they would be caused by the environment conditions.
\end{abstract}

\section{Introduccion}

El mejillón Perna perna (L) ha sido estudiado en Venezuela por varios autores desde diversos puntos de vista. Andreu (1962), comenzó la instalación de balsas de cultivo. Vélez \& Martinez (1967), efectuaron observaciones experimentales sobre su reproducción y desarrollo larval. Carvajal (1969) analizó la fluctuación cuantitativa mensual de las larvas, el crescimiento y las condiciones ambientales. Benitez (1968), Benitez \& Okuda (1971) determinaron la variación estacional de la composición química del mejillón cultivado y natural respectivamente. Vélez (1971) comparó el indice de engorde entre el mejillón natural y cultivado. Arocha (1974) estudió el efecto de la profundidad sobre el ren. dimiento del cultivo y la composición química durante el creci. miento del mejillón. Mandelli \& Acuña (1975) realizaron un análisis de las áreas y técnicas de cultivo del mejillón.

El Centro de Investigaciones Científicas de la Universidad de Oriente, ha utilizado la Bahía El Guamache (Isla de Margari ta) para el cultivo de mejillón, pero el conocimiento que se tenía de las condiciones ambientales era solo muy general y basada en los trabajos de Richards (1960), Gade (1961), Ljöen \& Herrera (1965), Griffiths \& Simpson (1972), Okuda (1974) y otros autores, que incluían la zona de la Isla de Margarita, pero ninguno era tan local y costero para considerar la Bahía El Guamache.

Como un complemento al programa de cultivo en balsas. se programó un estudio para conocer si el lugar ofrecía buenas condiciones ambientales y determinar las variaciones de la composición química, peso total, peso carne, peso concha y agua intervalvar del meiillón en cultivo.

\section{Materiales y Metodos}

En una de las balsas de cultivo de mejillones, se sembraron cuerdas para realizar exclusivamente el estudio de ciertas variables del mejillón en crecimiento. Se adoptó la cuerda comercial de 7 metros de largo, pero sembrando solamente una sección de un metro en cada uno de los siguientes intervalos de profundidad: superricie $(\mathrm{U}-1 \mathrm{~m})$, media $(3-4 \mathrm{~m})$ y tondo $(6-7 \mathrm{~m})$, dejando el resto de la cuerda sin mejillón a fin de facilitar el muestreo, el que se realizó conjuntamente con el de variables ambientales en forma quincenal desde Julio de 1975 a Junio de 1976. Además se incluyen 12 meses adicionales de observaciones de temperatura, salinidad y oxígeno.

Se tomaron dos tipos de muestras, una correspondiente al intervalo de tallas $65-70 \mathrm{~mm}$, constante para el periodo de muestreo, que duró solamente 9 meses, debido a que posteriormente no se obtuvieron ejemplares de esa longitud. Las primeras muestras se tomaron de las cuerdas comerciales sembradas por el programa de cultivo, hasta que hubo mejillones de esa talla en las sembradas para ese estudio. La talla de los mejillones'de la otra muestra se tue aumentanav em $5 \mathrm{~mm}$ de longitud cada mes, comenzando desde la talla de siembra $(40-45 \mathrm{~mm})$ hasta la de 100 $-105 \mathrm{~mm}$ ( 13 meses).

Los mejillones fueron processados siguiendo fundamentalmente la técnica de Fraga (1955). Los ejemplares fueron pesados para obtener el peso total y luego abiertos y colocados sobre una superficie inclinada aurante 10 minutos, para que escurriera el aqus intervalar y nuevamente pesados para determinar el peso del agua. Posteriormente se separó la carne, incluyendo los músculos aductores, se colocó en cápsulas de Petri taradas y se pesaron ambas partes para obtener el peso carne y peso concha. La carne fue secada en estufa a $110^{\circ} \mathrm{C}$ hasta peso constante, para obtener la humedad por perdida de peso. Las cenizas fueron determinadas por calcinación en horno a $550^{\circ} \mathrm{C}$ durante 24 horas. Las proteinas se determinaron por el método Kjeldahl semimicro (Fraga, 1955). Los valores de nitrogeno fueron convertidos en proteina multiplicándolos por un factor de 6,25 . La cantiaau de oxígeno disuelto, nitrito, nitrato, amonio y fosfato fueron determinados siguiendo las técnicas descritas por Strickland \& Parsons (1972). La salinidad se determinó con un salinómetro de inducción. La clorofila fue analizada por el método de SCOR/ /UNESCO (1969). La cantidad de materia en suspensión fue medida filtrando la muestra de agua a través de un filtro tarado de membrana Millipore HA, secado hasta peso constante y constituyendo la diferencia de pesada la cantidad de materia en suspensión.

\section{Analisis de los Resultados}

\section{Condiciones Ambientales}

Las variaciones anuales de los parámetros ambientales básicos temperatura y salinidad, mostraron ciertas características que pueden relacionarse con las condiciones meteorológicas, que imperaron en la zona durante el tiempo que duro el estudio. La temperatura del agua (Fig. 1), siguió un patrón intimamente ligado a las variaciones de la temperatura del aire y logicamente relacionadas con los otros parámetros metereológicos, que alteran la temperatura ambiental, como la insolación y la cantidad de radiación solar. $\mathrm{La}$ temperatura alcanzó el máximo entre los meses de Septiembre v Noviembre y el mínimo entre Febrern y Abril. con un valor medio para todo el periodo de $25,05^{\circ} \mathrm{C}$ (Tab. 1).

Entre Julio y Noviembre de 1975, la salinidad (Fig. 2), se presentó bajo el promedio de los 24 meses $(36,46 \%$ oo), a pesar de corresponder a la época de más calor y de mayor evaporación del año. Esto se explicaría por la gran cantidad de precipitación caída entre los meses de Mavo v Noviambre de 1975, como lo demuestran los $452,8 \mathrm{~mm}$ (Tab. II), sienao que para la zona la media anual es de $276 \mathrm{~mm}$ (Contreras, 1967). Este aporte de agua dulce, determinó que la salinidad descendiera baio el promedio, pero no ocurrió lo mismo en igual período del año 1976, donde la salinidad fue ligeramente superior al valor medio, pués, ahora la precipitación fue casi un cuarto de la caída en Mayo-Noviembre de 1975 y la evaboración e insolación fueron superiores (1 ab. II), lo que justiticaria que los valores de salinidad fueron más altos que los del año anterior. El mismo fenómeno es reportado por Pinela \& Aguado (1977) en la Banía de Mangle, lugar cercano a la Bahía El Guamache. En cuanto a los dos períodos Diciembre-Mayo, la salinidad estuvo generalmente por sobre el promedio, a pesar de ser la época en que la temperatura del agua y aire es más baja.

La Bahía El Guamache mostró una buena oxigenación, con valores de saturacion siempre por sobre el $81 \%$ (Tab. 1). $\mathrm{La}$ distribución a lo largo del año fue relativamente uniforme, pero se apreció un leve incremento en la cantidad de disolución que posee el agua, causada fundamentalmente por el descenso de temperatura.

Los vajores ue tosrato usuerto permanecieron durante todo el año generalmente bajo $0,5 \mu \mathrm{g}$-at $/ \mathrm{L}$, con un promedio de 0,21 in g-at/L (Tab. III), lo que puede considerarse normal para agua costera, donde la actividad fotosintética remueve nutrientes. En cuanto al nitrógeno inorgánico, la forma nitrato fue relativamente constante a lo largo del año, estando por lo general bajo los 2 , i g-at $/ \mathrm{L}$ y con una mínima diferencia entre los tres niveles (Fig. 3, nivel medio). Los valores bajos de nitratos se debieron posiblemente al contínuo consumo, ya que el nivel de clorofila nunca descendió a valores críticos, lo que indica, que hubo una contínua utilización de este nutriente y la velocidad de renovación fue incapaz de producir un incremento apreciable de él en la columna de agua estudiada. Por su parte el nitrito presentó la menor fracción del nitrógeno inorgánico, estando muy cercano 


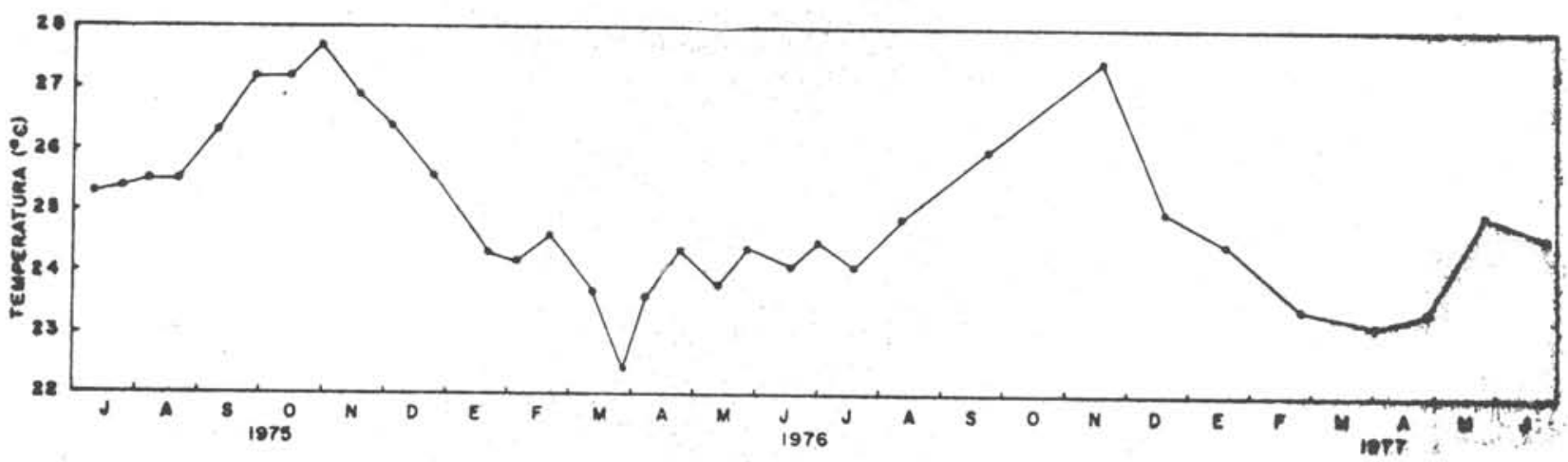

Fie. I Veriedidn meneval dal promedio de temperatura en le columna de aguo de 0-7 m.

TABLA I

PROMEDIOS Y VALORES DE TEMPERATURA, SALINIDAD Y OXIGENO PARA EL PERIODO JULIO 1975 a JUNIO 1977

\begin{tabular}{|c|c|c|c|c|c|c|c|c|c|c|c|c|}
\hline \multirow[t]{2}{*}{ PROF.(m) } & \multicolumn{3}{|c|}{ TEMPERATURA $\left({ }^{\circ} \mathrm{C}\right)$} & \multicolumn{3}{|c|}{ SALINIDAD $(\% \circ)$} & \multicolumn{3}{|c|}{ OXIGENO(ml/L) } & \multicolumn{3}{|c|}{$\begin{array}{l}\text { SATURACION } \\
\text { OXIGENO (\%) }\end{array}$} \\
\hline & Max. & Min. & Prom. & Max. & Min. & Prom. & Max. & Min. & Prom. & Max. & Min. & Prom. \\
\hline 0 & 27,80 & 22,40 & 25,11 & 37,77 & 35,25 & 36,39 & 4,94 & 3,79 & 4,35 & 100 & 81 & 93 \\
\hline 3.5 & 27,70 & 22,40 & 25,03 & 37,77 & 35,68 & 36,47 & 5,08 & 3,79 & 4,36 & 100 & 81 & 94 \\
\hline 7 & 27,70 & 22,40 & 25,01 & 37,83 & 35,75 & 36,51 & 4,96 & 3,79 & 4,34 & 100 & 81 & 93 \\
\hline $\begin{array}{l}\text { Promedio } \\
0-7 \mathrm{~m}\end{array}$ & - & - & 25,05 & - & - & 36,46 & - & - & 4,35 & - & - & 93 \\
\hline
\end{tabular}

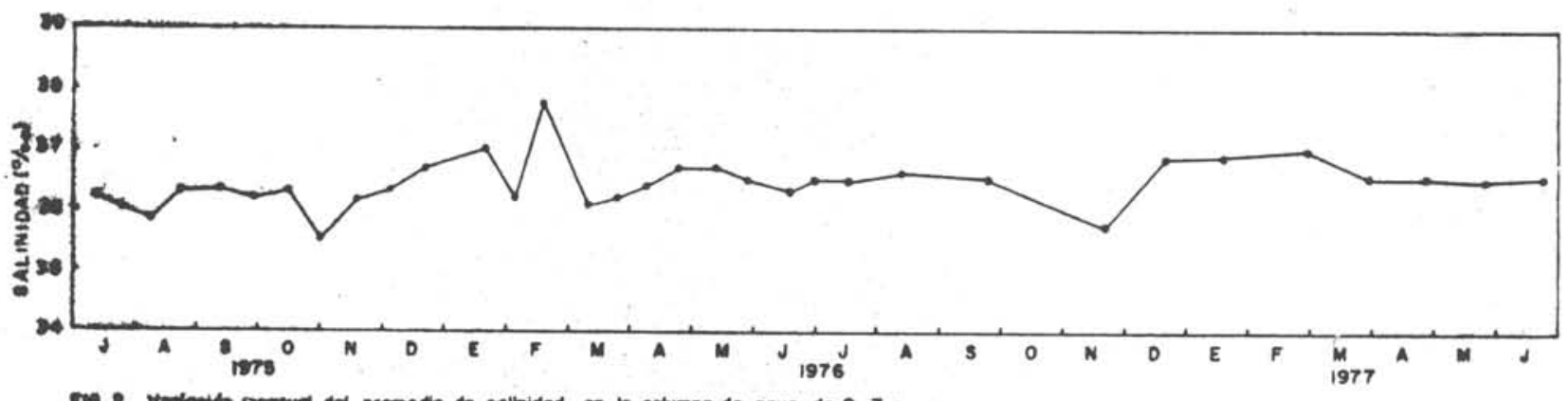

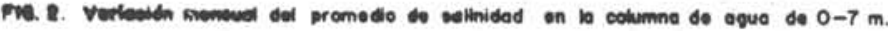

TABLA II

DATOS METEOROLOGICOS DE LA ESTACION PUNTA DE PIEDRAS PARA EL PERIODO

JUNIO 1975 A MAYO 1977

\begin{tabular}{lccrr} 
& \multicolumn{3}{c}{ PERIODO } \\
JUNIO - NOV. & DIC. - MAYO & JUNIO - NOV. & DIC. - MAYO \\
VARIABLE & $1975-1975$ & $1975-1976$ & $1976-1976$ & $1976-1977$ \\
EVAPORACION $(\mathrm{mm})$ & $1.613,70$ & $1.500,40$ & $1.810,10$ & $1.901,10$ \\
PRECIPITATION $(\mathrm{mm})$ & 452,80 & 259,40 & 120,50 & 138,60 \\
INSOLACION & $1.554,80$ & $1.454,30$ & $1.635,60$ & $1.690,20$
\end{tabular}




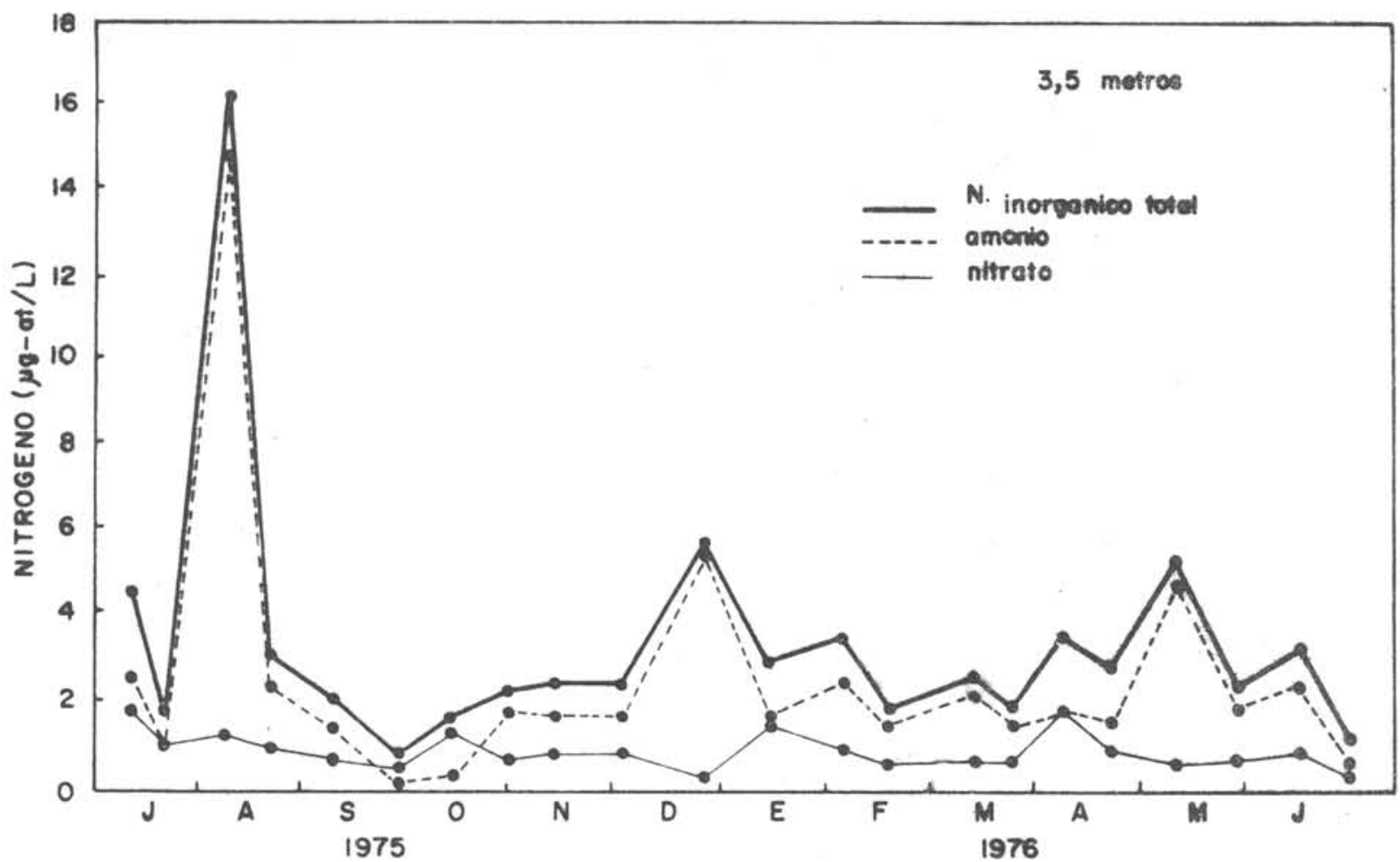

Fig. 3 - Variación mensual de nitrato, amonio y nitrógeno inorganico total en $3,5 \mathrm{mts}$.

TABLA III

PROMEDIOS Y VALORES EXTREMOS DE AMONIO, NITRITO, NITRATO Y FOSFATO PARA EL PERIODO JULIO 1975 A JUNIO 1976.

PROF. (m) AMONIO $(\mu \mathrm{g}$-at N/L)

$\begin{array}{rrrrrrr} & \text { Max. } & \text { Min. } & \text { Prom. } & \text { Max. } & \text { Min. } & \text { Prom. } \\ 0 & 15,08 & 0,20 & 2,82 & 0,29 & 0,00 & 0,08 \\ 3.5 & 15,02 & 0,26 & 2,24 & 0,29 & 0,00 & 0,07 \\ 7 & 7,22 & 0,46 & 2,22 & 0,38 & 0,00 & 0,09\end{array}$

a cero. La mayor cantidad de este nutriente estuvo representada por el amonio, que además fue el más irregular en sus valores y el que realmente provocó algunos máximos en la curva de variación de nitrógeno inorgánico total (Fig. 3).

En la clorofila (Fig. 4), sobresalen algunos máximos en Julio-Agosto, Octubre-Noviembre, Abril-Mayo y Junio de 1976 con una diferencia mínima entre los tres niveles, salvo en Jutio de 1975 y Junio de 1976, en que el valor más alto fue el de 7 merros y el menor correspondió a la superficie. Hubo un lapso desde Diciembre a Marzo, donde la clorofila se mantuvo bajo $1 \mu \mathrm{g} / \mathrm{L}$, correspondiendo a la época invernal cuando la actividad fotosintética es menor. Por su parte la materia en suspensión (F g. S), no guarao una relacion clara con a cannuad de clorofila, mostrando más bien la mayor cantidad en la época de más lluvia, lo cual se debería al aporte de materia sólida desde tierra por el agua de escorrentía y la remoción del fondo, por acción de un mayor movimiento del agua causado por el viento.

\section{Variables del mejilión}

El peso carne de la talla $65-70 \mathrm{~mm}$ (Fig. 6), presentó los valores más altos en el nivel superficial $(0-1 \mathrm{~m})$, exceptuándose los meses de Septiembre y Octubre en donde el valor máximo se dió en la profundidad media $(3-4 \mathrm{~m})$. Los valores de Enero y Marzo son altos comparados con el resto de los resultados, correspondiendo con el período de más baja temperatura del agua y de salinidad relativamente más alta. Por otra parte, los resultados más bajos de superficie se obtuvieron en los meses de Septiembre y Octubre, cuando la temperatura estaba alta y la salinidad por debajo del promedio anual. Los valores más bajos no
NITRATO ( $\mu g$-at N/L)

FOSFATO $(\mu \mathrm{g}-\mathrm{at} \mathrm{P} / \mathrm{L})$

Max. Min. Prom.

Max. Min. Prom.

2,02

$0,21 \quad 0,87$

$0,40 \quad 0,00$

0,21

1,83

$0,42 \quad 0,80$

$0,55 \quad 0,00$

$2,50 \quad \cdot 0,20 \quad 0,85$

$0,50 \quad 0,00$

0,18

son coincidentes para los tres nrveles, mientras en superficie se registran en Septiembre y Octubre, en las otras tres profundidades fueron en Diciembre-Enero, cuando la cantidad de clorofila fue menor y la temperatura estaba baja, Los resultados de los dos niveles más profundos concuerdan con los de Benitez (1968) para el Estado Sucre. En los niveles 3-4 m y 6-7 m el peso carne fue superior entre Octubre y Noviembre, correspondiendo con un período de florecimiento de fitoplancton. En lineas generales se apreció una buena correlación entre aumento de peso y mayor cantidad de clorofila.

El peso de la concha, fluctuó entre 8,88 y $11,00 \mathrm{~g}$ (Tab. IV), con una leve tendencia a descender gradualmente con el tiempo en los tres niveles de muestreo y con una variación irregular respecto a ta profundidad. El peso ael agua intervalvar, mostró un aumento en los primeros tres meses (Agosto-Octubre) con poca diferencia entre los valores de las tres profundidades, luego se produjo una estabilización temporal, para posteriormente volverse irregular. Los valores extremos del agus intervalvar fueron 2,64 y $6,83 \mathrm{~g}$ entre los cuales tluctuaron los pesos durante el período de observación.

El mínimo de agua en la talla $65-70 \mathrm{~mm}$, se presentó en el mes de Agosto para las tres profundidades. El contenido de cenizas también registró su valor más bajo en este mes y el máximo en Febrero-Marzo (Fig. 7). con buena correspondencia con los resultados reportados por Benitez (op. cit) para el mejillón cultivado en Playa Grande, Estado Sucre. En los muestreos de la talla progresivamente superior el contenido de agua y cenizas (Fig. 8), mostraron los valores mínimos en Agosto de 1975 (talla $45-50 \mathrm{~mm}$ ) y Julio de 1976 (Talla 100-105 mm.). Estas curvas de humedad y cenizas siguieron la misma tendencia que las de 


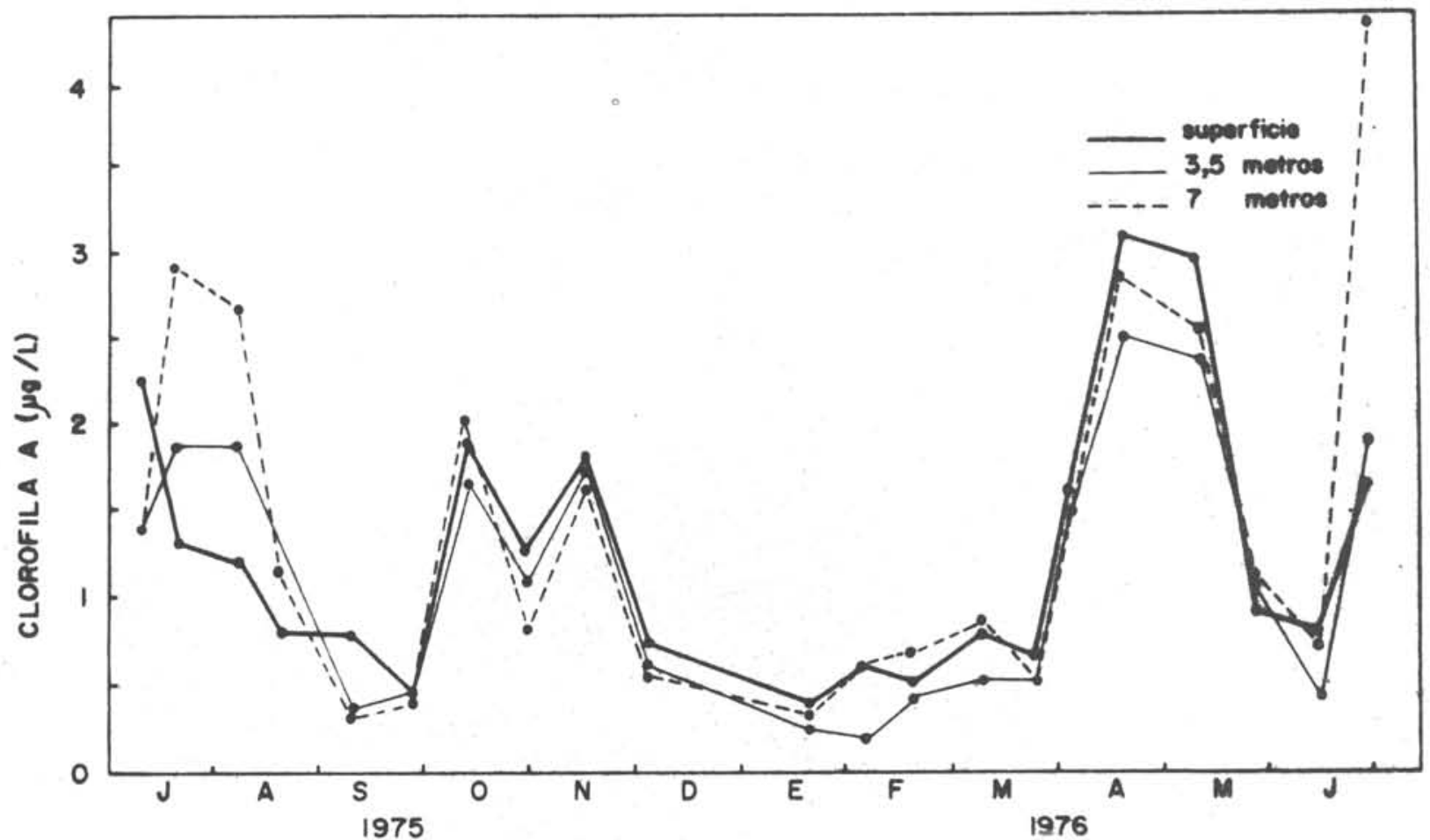

Fig. 4 -Variación mensual de clorofila "a" en superficie, 3, 5 y 7 metros.

TABLA IV

VALORES EXTREMOS Y PROMEDIOS DEL PESO CARNE,
PESO CONCHA, PESO AGUA INTERVALVAR PARA EL INTERVALO DE TALLAS $65-70 \mathrm{~mm}$

$\begin{array}{lccc}\text { VARIABLE } & \text { MINIMO } & \text { MAXIMO } & \text { PROMEDIO } \\ & \mathbf{g} & \mathbf{g} & \mathbf{8} \\ \text { Peso Carne } & 8,05 & 10,87 & 9,15 \\ \text { Peso Concha } & 8,88 & 11,00 & 9,65 \\ \text { Agua Intervalvar } & 2,64 & 6,83 & 4,78\end{array}$

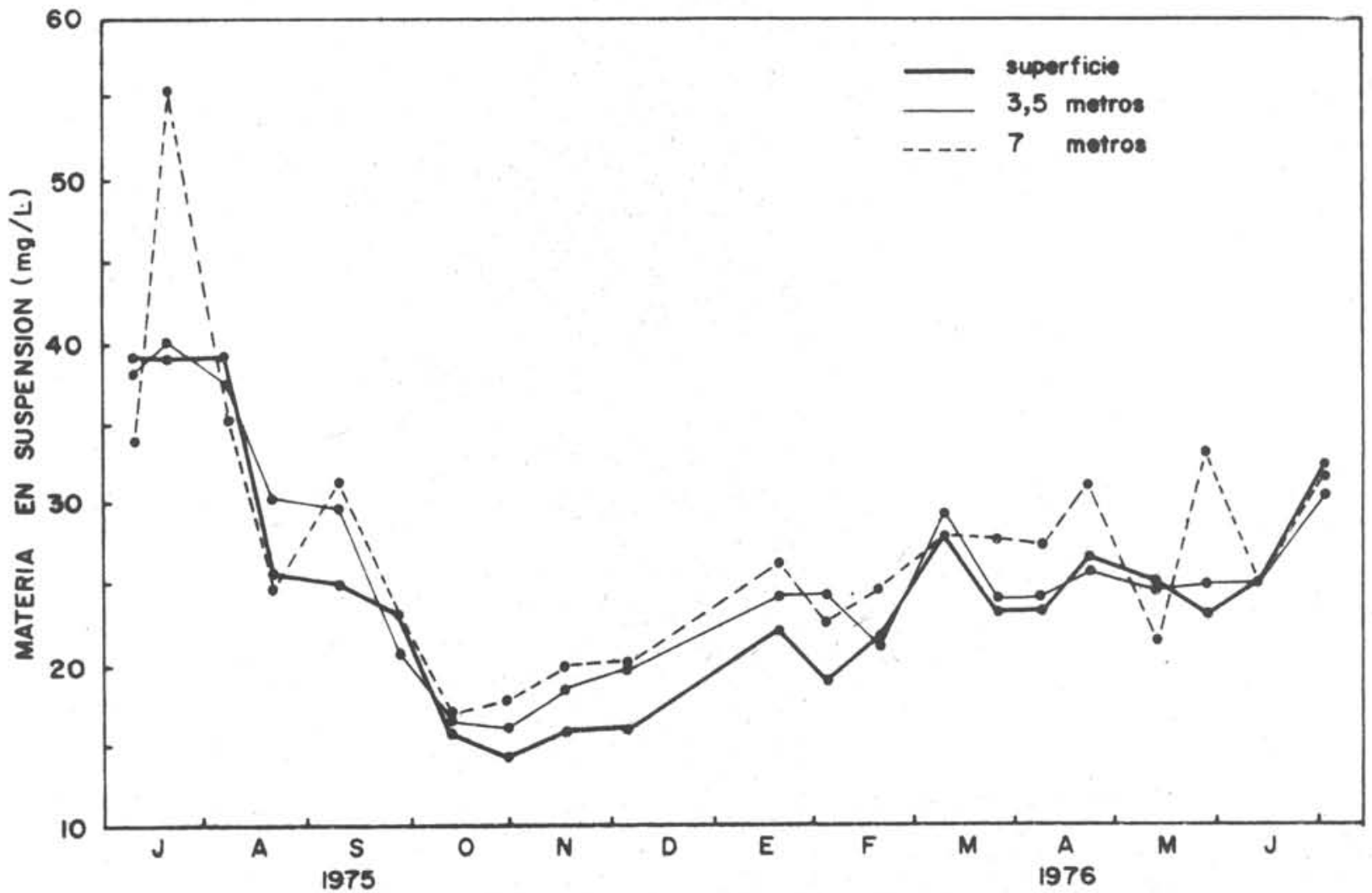

Fig. 5 - Variacion mensual de materia particulada en suspensón en superficie, 3, 5 y 7 metros. 


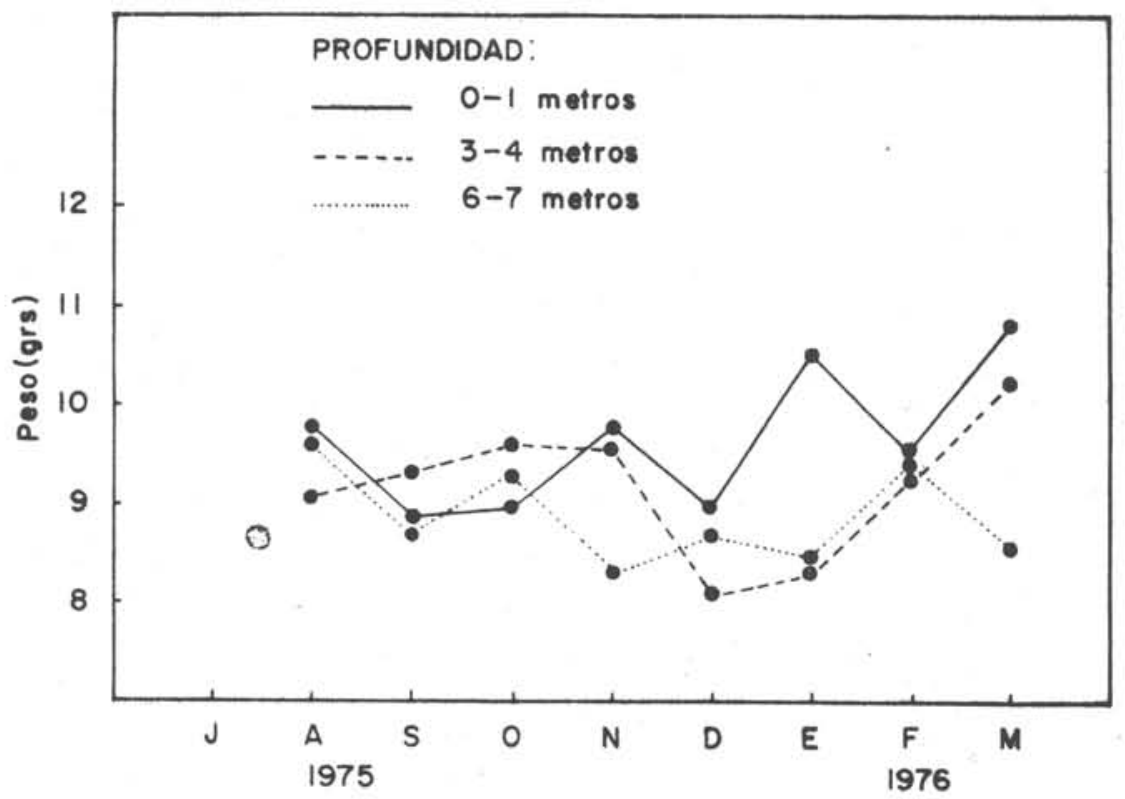

Fig. 6 - Variación del peso de la carne de la talla $65-70 \mathrm{~mm}$. en las tres profundidades estudiadas.
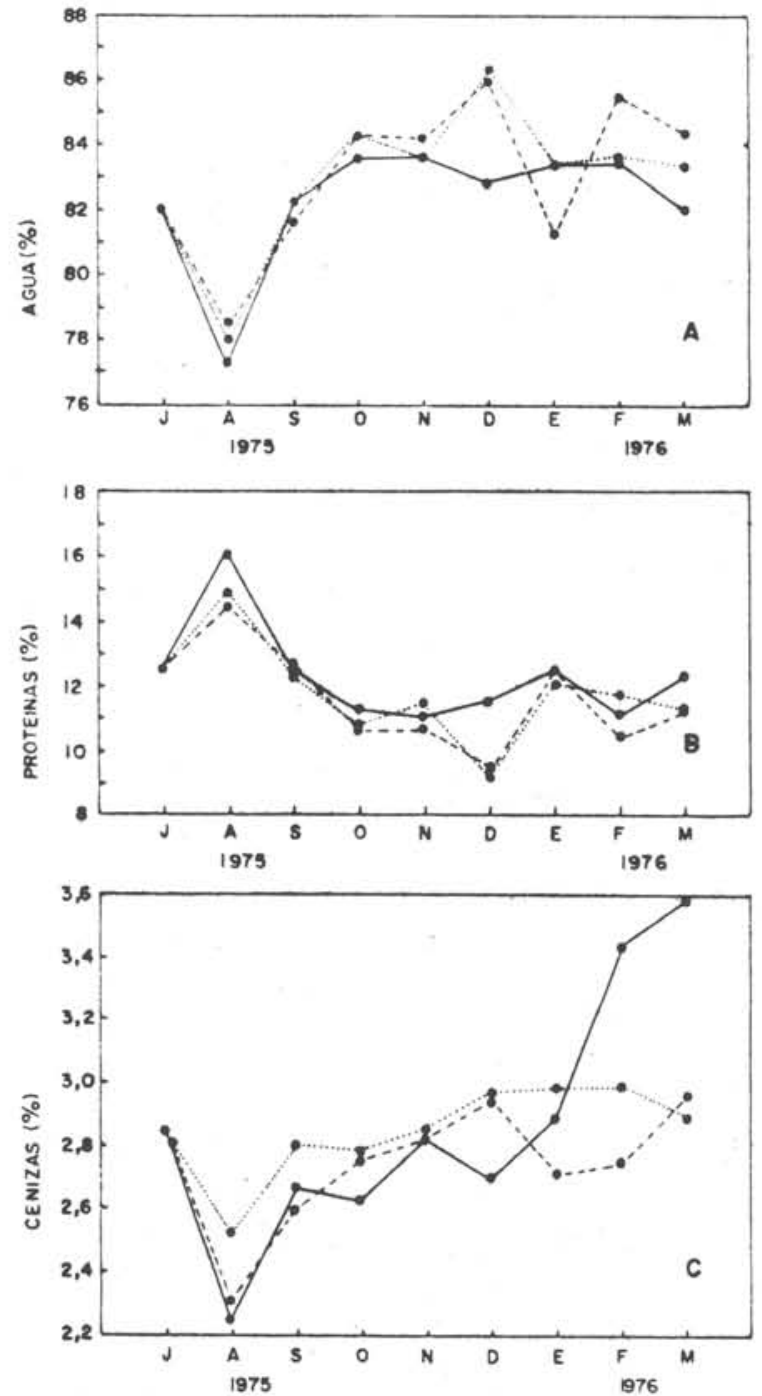

Fig. 7 - Humedad, proteinas y cenizas en el mejillon del intervalo de tallas $65-70 \mathrm{~mm}$. superfície, - - $-3-4 \mathrm{~m}, \ldots \ldots 6-7 \mathrm{~m}$. 


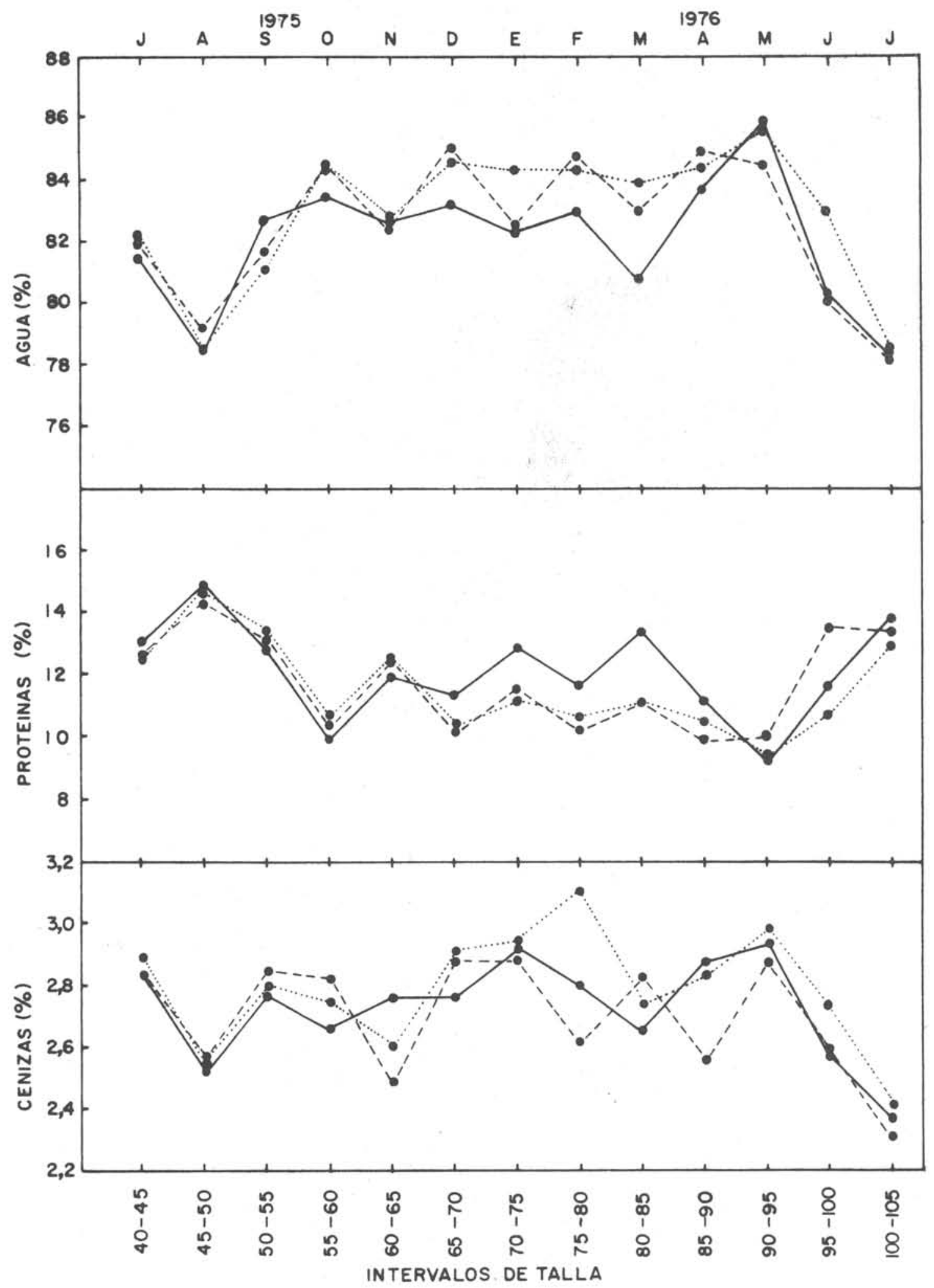

Fig. 8 - Humedad, proteinas y cenizas en el mejillón desde el intervalo de talla $40-45$ al 100-105 mm. superfície, $-3.4 \mathrm{~m}$ 6-7 m.

la talla 65-70 mm (Julio 75-Marzo 76), de lo que se deduce que en estas dos variables no influyen fundamentalmente la talla y son más bien las condiciones ambientales las que estarían causando las diferencias estacionales.

El contenido de proteinas de la talla $65-70 \mathrm{~mm}$ (Fig. 7), mostró su valor máximo en Agosto, lo cual también concuerda con los resultados de Benitez (op. cut.). Por ora parte, Benitez \& Okuda (1971) señalaron un máximo de proteínas en Diciembre para Puerto Santo. Estado Sucre y Arocha (1974) máximos para Febrero-Marzo y Julio de 1974 en la Bahia de Mangle (İsia de Margarita). Este último máximo tendría relación con el que se reporta en este trabajo para Agosto, ya que son lugares muy cercanos. Arocha cita, que el contenido de proteınas fue superior en los mejillones sembrados más profundos, esto no se ajusta con nuestros resultados, ya que generalmente el valor en el nivel superficial fue superior al resto, datos que son coincidentes con los de Benitez (1968). Esta diferencia es probable que se deba a las condiciones propias de la Bahía de Mangle (entrada de la laguna La Restinga), donte Arocha (op. cit.), efectuó su trabajo. La curva de cantidad de proteínas de las muestras de talla progresivamente superior (entre $40-85 \mathrm{~mm}$ ), son muy semejantes a la de la talla $65-70 \mathrm{~mm}$, por lo tanto, en el contenido de proteínas tampoco influye la talla y son también las condiciones ambientales y de alimentación las que determinan las diferencias 
estacionales. Comparando las curvas de variación de humedad, cenizas y proteínas de ambos tipos de muestreos, se aprecia claramente una relación inversa entre la humedad y cenizas, con respecto a la cantidad de proteínas. Por otra parte, los valores más altos de proteína se producen inmediatamente después de un máximo de clorofila.

\section{Conclusiones}

La temperatura y salinidad siguieron un patrón de variación en estrecha relación, con los parámetros meteorológicos que imperaron en la zona durante el estudio. La capa de agua de 0 a 7 metros, mostró una buena oxigenación con valores de saturación de oxígeno por sobre el $81 \%$. Los nutrientes primarios mostraron un nivel aceptable para agua costera, sobresaliendo el amonio como el más abundante. La clorofila se mantuvo entre 0,25 y $4.50 \mu \mathrm{g} / \mathrm{L}$. Se podría concluir, que desde el punto de vista de las variables ambientales, la Bahía El Guamache ofrece buenas condiciones para el cultivo de mejillones en escala comercial.

De los resultados obtenidos en las muestras de mejillones, se deduce, que el contenido de agua, cenizas y proteínas varió independientemente de la talla y son las condiciones ambientales las que causan las diferencias (variables hidrológicas y de alimentación). La humedad y las cenizas presentaron los valores más bajos, cuando el contenido de proteínas fue más alto y fueron subiendo, a medida que el contenido proteíco bajaba. A su vez. los valores más altos de proteína, por lo general correspondieron a la superficie.

\section{Referencias Bibliográficas}

ANDREU, B. 1962. La mitilicultura y sus perspecavas en costns orientales de Venezuela. Cienc. al día, (12):86-88.

AROCHA, P. 1974. Efecto de la profundidad de cultivo y las condiciones ambientales sobre la variación de la composición química del mejillón (Perna perna) durante su crecimiento. Trabajo de Ascenso, Universidad de Oriente, Venezuela.

BENITEZ, J. 1968. Variación mensual de la composición quimica del mejillón Perna perna (L.). Boln Inst. oceanogr. Univ. Oriente, $7(1): 137-147$.
\& OKUDA, T. 1971. Variación estacional en la composición química del mejillón Perna perna (L.) natural. Boln. Inst. oceanogr. Univ. Oriente, 10(1):3-8.

CARVAJAL, J. R. 1969. Fluctuación mensual de las larvas y crecimiento del mejillón Perna perna y las condiciones ambientales de la ensenada de Guatapanare, Edo. Sucre, Venezuela. Boln Inst. oceanogr. Univ. Oriente, $8(1 / 2)$; 13-20

CONTRERAS, A. 1967. Características pluviométricas del Estado Nva. Esparta. Mems. Soc. Cienc. nat. La Salle, 27176) 46-75.

FRAGA, F. 1955. Variación estacional de la composición quí. mica de la anchoa (Engraulis encrasicholus). Investigación pesq., (2):109-125

SEIFFITHS, R. C. \& SIMPSON, J. G. 1972. Afloramiento y otras caracteristicas oceanográficas de aguas costeras del Nordeste de Venezuela. Sér. Recursos Explot. Pesq., 2(4)

LJOEN, R. \& HERRERA, L. E. 1965. Some oceanographic conditions of the coastal of Eastern Venezuela. Boln Inst. oceanogr. Univ. Oriente, 4(1):7-50.

MANDELLI, E. \& ACUÑA, A. 1975. The culture of the mussel, Perna perna, and mangrove oyster, Crassostrea rhizophorae, in Venezuela. Mar. Fish. Rev., 37(1):15-18.

OKUDA, T. 1974. Características oceanográficas generales de la costa Sur-oriental del Mar Caribe. Cuadernos azules, III Conferencia de las Naciones Unidas sobre Derecho del Mar, Caracas.

PINEDA, J. \& AGUADO, A. 1977. Clororua y eiclo de nutrientes en la laguna La Restinga. (no publicado).

RICHARDS, F. A. 1960. Some chemical and hydrographic observations along the north coast of South America. 1. Cabo Tres Puntas do Curacao, including the Cariaco Trench and Gulf of Cariaco. Deep Sea Res., 7(3):163-182.

SCOR/UNESCO Group. 1969. Determination of photosynthetic pigments in sea-water. Monogr. oceanogr. Methodol., (1): $1-66$.

STRICKLAND, J. D. H. \& PARSONS, T. R. 1972. A practical handbook of seawater analysis. Bull. Fish. Res. Bd Can., (167):1-311. 